\title{
Interfacial operator approach to computing band structures for photonic crystals of polar materials
}

\author{
Ruey-Lin Chern, ${ }^{1, *}$ Chien C. Chang, ${ }^{1,2, \dagger}$ and C. Chung Chang ${ }^{2}$ \\ ${ }^{1}$ Institute of Applied Mechanics, National Taiwan University, Taipei 106, Taiwan, Republic of China \\ ${ }^{2}$ Division of Mechanics, Research Center for Applied Sciences, Academia Sinica, Taipei 115, Taiwan, Republic of China
}

(Received 24 January 2006; revised manuscript received 20 April 2006; published 27 June 2006)

\begin{abstract}
In this study, we propose an interfacial operator approach to compute surface phonon modes for one- and two-dimensional periodic arrays of polar materials in a finite-difference formulation. The key aspect of the approach is to introduce an interfacial variable along the interface between the polar material and the surrounding dielectric material, which represents the local strength of the surface phonon modes along the interface. In this approach, the apparently nonlinear eigenvalue problem can be reformulated as a quadratic eigensystem, and thus further reduced to a standard linear eigenvalue problem. Band structures can be computed directly without the need of examining transmission spectra as in the finite-difference time-domain method, or locating the mode frequency by testing an auxiliary function in other methods. Applying the method to four different types of photonic crystals of polar materials, we are able to uncover several interesting results by studying the effect of dimension, the size (filling ratio) effect, the effects of the transverse optical phonon frequency $\left(\omega_{T}\right)$, and longitudinal optical phonon frequency $\left(\omega_{L}\right)$ as well as the effect of shape or geometry of the polar material.
\end{abstract}

DOI: 10.1103/PhysRevB.73.235123

\section{INTRODUCTION}

Photonic crystals made of dielectric materials have been extensively studied since 1987., ${ }^{1,2}$ The most distinguished feature is their full band gaps, where periodicity of the structure provides a scattering mechanism to prohibit propagation of the electromagnetic field from all directions over certain ranges of frequencies. If the dielectrics are replaced by perfect metals, ${ }^{3-9}$, the fields are completely expelled from the metals and vanish inside. As a result, large band gaps can be opened up. If more realistic dispersive metals replace perfect conductors in the photonic structures, ${ }^{10-15}$ collective motion of free electrons gives rise to resonance in optical properties. In particular, surface plasmon modes may appear in the optical frequency range, and therefore, these structures are also named plasmonic crystals. ${ }^{16,17}$ On the other hand, if polar materials replace the metals, ${ }^{18-21}$ surface phonon modes may result from the coupling of lattice vibration of the ionic structure and the electromagnetic field. In the meanwhile, densely distributed resonant cavity modes as well as other modes also arise in these structures. This type of periodic structures are termed as photonic crystals of polar materials or simply polaritonic crystals. ${ }^{21}$

The model for polar materials considered in the present study is given by ${ }^{22}$

$$
\varepsilon(\omega)=\varepsilon_{\infty}\left(\frac{\omega^{2}-\omega_{L}^{2}}{\omega^{2}-\omega_{T}^{2}}\right),
$$

where $\varepsilon_{\infty}=\varepsilon(\infty)$ is the dielectric constant at a very high frequency, $\omega_{T}$ is transverse optical (TO) phonon frequency, and $\omega_{L}$ is the longitudinal optical (LO) phonon frequency, which is related to $\omega_{T}$ through the Lyddane-Sachs-Teller (LST) relation $\omega_{L}^{2} / \omega_{T}^{2}=\varepsilon(0) / \varepsilon(\infty)$. It is known that a polar material does not support propagating modes with frequencies inside the polariton gap $\left(\omega_{T}<\omega<\omega_{L}\right)$, while the bulk modes with frequencies outside the gap follow an anticrossing scheme. The situation changes drastically if we consider interfaces
PACS number(s): 78.20.Bh, 42.70.Qs, 02.70.-c, 02.70.Bf

between polar materials and other dielectrics. The simplest interface is a plane between the polar material and a surrounding dielectric. In this case, there exists the surface phonon mode with the frequency inside the polariton gap. Let $\varepsilon_{d}$ be the dielectric constant of the surrounding medium. At the large wave number limit, the frequency of the surface phonon mode approaches

$$
\omega_{\text {sph }}=\sqrt{\frac{\varepsilon_{\infty} \omega_{L}^{2}+\varepsilon_{d} \omega_{T}^{2}}{\varepsilon_{\infty}+\varepsilon_{d}}}
$$

which is called the surface phonon frequency. Furthermore, a periodic array of polar materials may fold the bands within the first Brillouin zone and produce extra bands not observed in a simple interface.

There is a large difference between the dielectric photonic crystals and those made of metals. For dielectrics, the electric field produces a polarization in the same direction, and the band structures are scale invariant. For dispersive metals, the electric field produces an adverse polarization which may result in a negative dielectric constant due to the electron motion at frequencies below the plasma frequency $\omega_{p}$. The band structures are no longer scale invariant because of the characteristic $\omega_{p}$ of the metal. For arrays of polar materials, oscillation of the effective charges of the ions due to lattice vibration results in two distinct classes of resonant modes. One class of modes are analogous to the case of surface plasmons in dispersive metals, but usually occurs in the infrared frequency range. These waves are surface phonons or surface phonon modes. The other are resonant cavity modes or waveguide modes corresponding to very large dielectric constants lying immediately below $\omega_{T}$.

It has been difficult to compute eigenmodes and band structures of frequency-dependent materials, in particular, for transverse electric (TE) modes. The major difficulty comes from the apparently nonlinear formulation of the eigenvalue problem. ${ }^{23}$ Several approaches have been proposed to study this problem, including plane wave expansion method ${ }^{20}$ and 


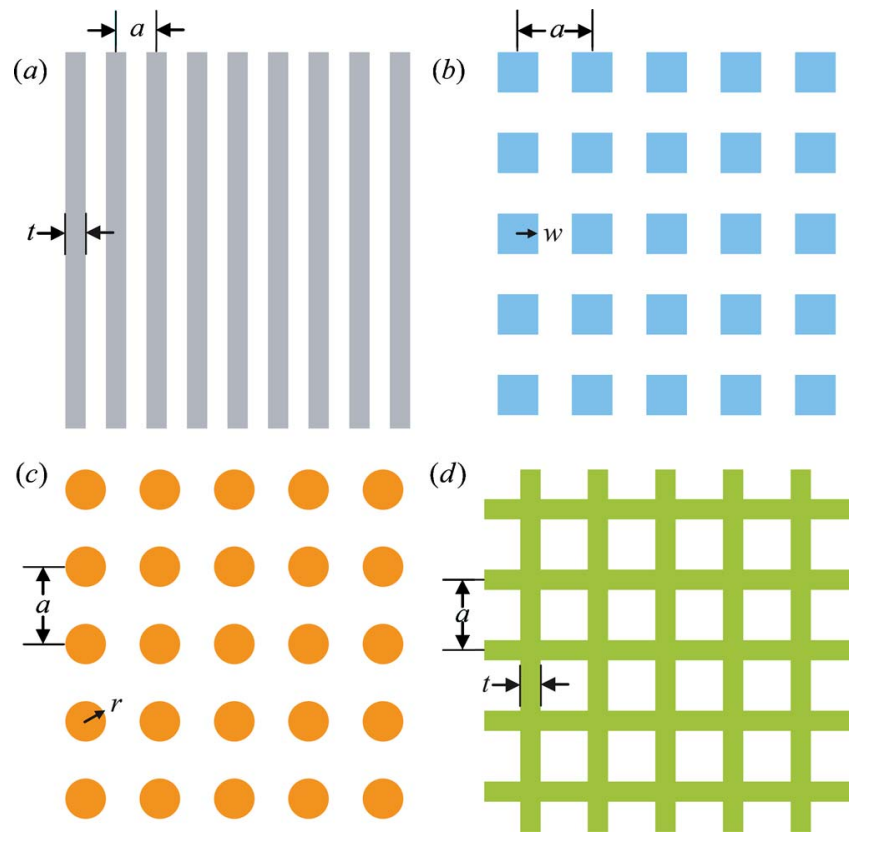

FIG. 1. (Color online) Plasmonic crystals made of dispersive metals. (a) 1D layered structure, (b) 2D array of square cylinders, (c) 2D array of circular cylinders, and (d) 2D array of grid cylinders.

vectorial eigenmode expansion method. ${ }^{21}$ Other approaches such as transfer matrix method, ${ }^{18}$ layer-Korringa-KohnRostoker method, ${ }^{24}$ multiple multipole method, ${ }^{15}$ and finitedifference time-domain method ${ }^{14}$ can also be applied to frequency-dependent problems. Another difficulty comes from the special physical features of the solution. Localized surface plasmons for arrays of dispersive metal, as well as surface phonons for arrays of polar material, require very fine resolution schemes. An even more critical situation is that the dielectric function changes sign across the dielectricmetal or dielectric-polar material interface over some frequency ranges. This will cause the change of type of the eigensystem and may induce numerical instability in solving the eigenvalue problem.

In one of our previous papers, ${ }^{25}$ we developed an interfacial operator approach to compute band structures for photonic crystals of dispersive metals. In particular, we considered the free-electron and the Drude models for the metal property. In this approach, the apparently nonlinear eigenvalue problem can be reformulated as a linear eigensystem which is solved by standard eigenvalue solvers. In the present study, we extend this approach to be applicable to photonic crystals of polar materials with the dielectric function in Eq. (1). In this approach, we will obtain a quadratic eigensystem, which in turn can be reduced to a linear eigensystem at the expense of doubling the matrix size. Moreover, we can dispense with the need of examining transmission spectra as in the finite-difference time-domain method, or locating the mode frequency by testing an auxiliary function in other methods.

In the present study, we investigate the four types of polaritonic crystals in Figs. 1(a)-1(d). Special emphases are placed upon the frequency bands within the polariton gap in addition to the resonant cavity modes with frequencies lying immediately below $\omega_{T}$. The four different photonic crystals of polar materials enable examination of (i) the effect of dimension between Fig. 1(a) and Figs. 1(b)-1(d), (ii) the size effect or the filling ratio effect $(t / a, w / a, r / a)$, (iii) the effect of the intrinsic frequencies $\omega_{T}$ and $\omega_{L}$, as well as (iv) the geometric effect (different shapes of polar materials) between Figs. 1(b)-1(d). It would be helpful to summarize the main results here and refer to the following sections for more details.

(1) About the effect of dimension, it will be shown that while there are only two branches of surface phonon modes for one-dimensional arrays, our numerical results indicate that there is infinite number of surface phonon modes for two-dimensional crystals. However, resonant cavity modes are supported by polaritonic crystals in both dimensions.

(2) Regarding the effect of $\omega_{T}$ and $\omega_{L}$, it is of interest to see what would happen if we let $\omega_{T}$ go to zero and $\omega_{L}$ tend to infinity. As $\omega_{T}$ goes to zero, the resonant cavity modes disappear all together, and as $\omega_{L}$ goes to infinity the surface phonon modes cannot be found with finite frequencies. It is further argued according to the Rayleigh quotient that the frequency bands of the polaritonic crystal will converge upward to those of the same photonic crystal made of perfect conductors. For finite values of $\omega_{L}$, there are other modes existing within the polariton gap in addition to the surface phonon modes. The transition of one such typical mode as $\omega_{L}$ goes from finite values to infinity will be discussed.

(3) Concerning the size effect, it will be shown that if the filling ratio is large, the distribution of resonant cavity modes is more spread in frequency, and the bulk modes inside the polariton gap would be expelled more effectively out of the gap. On the other hand, if the thickness ratio becomes smaller, the bands of surface phonon modes around $\omega_{\mathrm{sph}}$ is broadening. It is also argued that anticrossing of band dispersion of resonant cavity modes is also a size effect as the cutoff frequencies for TM modes are dependent upon the filling ratio.

(4) Finally with the effect of shape and geometry, it will be shown that the bands of TE modes for the array of circular cylinders could completely fill in the polariton gap and does not allow full photonic band gap within it, while the bands of TE modes for the array of grid cylinders are relatively more flat, and thus allowing full photonic band gaps within the polariton gap. It is also shown and argued that the arrays of grid cylinders are more effective in lifting the degeneracy of surface phonon modes than the arrays of circular cylinders.

\section{INTERFACIAL OPERATOR APPROACH}

The time-harmonic wave equations for linear, isotropic and nonmagnetic materials in two dimensions are given by

$$
\begin{gathered}
-\frac{1}{\varepsilon} \nabla^{2} E=\left(\frac{\omega}{c}\right)^{2} E, \\
-\nabla \cdot\left(\frac{1}{\varepsilon} \nabla H\right)=\left(\frac{\omega}{c}\right)^{2} H
\end{gathered}
$$

for TM and TE modes, respectively. For periodic structures, it is sufficient to solve the problem on one unit cell along 
with Bloch's condition as the boundary condition

$$
\begin{aligned}
& E\left(\mathbf{r}+\mathbf{a}_{i}\right)=e^{i \mathbf{k} \cdot \mathbf{a}_{i}} E(\mathbf{r}), \\
& H\left(\mathbf{r}+\mathbf{a}_{i}\right)=e^{i \mathbf{k} \cdot \mathbf{a}_{i}} H(\mathbf{r}),
\end{aligned}
$$

where $\mathbf{k}$ is the wave vector and $\mathbf{a}_{i}(i=1,2)$ are the lattice translation vectors. For frequency-dependent materials, the eigensystem no longer has a standard format since the eigenvalue itself appears in the solution operator

$$
\mathcal{L}(\Lambda) \phi=\Lambda \phi
$$

where $\Lambda=\omega^{2} / c^{2}$ is the eigenvalue, and $\phi$ is the eigenfunction, which can be either the $E$ or the $H$ field. If we discretize Eq. (7) in a straightforward manner, for example, by a finitedifference scheme, we will obtain a nonlinear eigensystem

$$
\mathbf{A}(\Lambda) \mathbf{x}=\Lambda \mathbf{x}
$$

where $\mathbf{A}$ is the matrix system and $\mathbf{x}$ is the eigenvector. This is one type of nonlinear eigenvalue problem, that is, nonlinear in eigenfrequency. However, if the dielectric function of the material has an analytical form, we are able to reformulate the original nonlinear eigenvalue problem as a standard eigensystem. For example, the eigensystem for TM modes (3), applied with the dielectric function (1), can be written as

$$
-\nabla^{2} E=\varepsilon_{\infty}\left(\frac{\Lambda-\Lambda_{L}}{\Lambda-\Lambda_{T}}\right) \Lambda E
$$

or, rearranged in the following form:

$$
\left[\Lambda^{2}-\Lambda\left(\Lambda_{L}-\frac{1}{\varepsilon_{\infty}} \nabla^{2}\right)-\frac{\Lambda_{T}}{\varepsilon_{\infty}} \nabla^{2}\right] E=0,
$$

which is a quadratic eigensystem, with $\Lambda_{T}=\omega_{T}^{2} / c^{2}$ and $\Lambda_{L}$ $=\omega_{L}^{2} / c^{2}$. By introducing an auxiliary variable $E^{\prime}=\Lambda E$, Eq. (10) can be written as

$$
\left[\begin{array}{cc}
0 & \mathbf{I} \\
\frac{\Lambda_{T}}{\varepsilon_{\infty}} \nabla^{2} & \Lambda_{L}-\frac{1}{\varepsilon_{\infty}} \nabla^{2}
\end{array}\right]\left[\begin{array}{l}
E \\
E^{\prime}
\end{array}\right]=\Lambda\left[\begin{array}{l}
E \\
E^{\prime}
\end{array}\right] .
$$

This is a linear eigensystem with standard format, which can be solved by standard eigenvalue solvers at the expense of doubling the matrix size. However, this could not be done for the TE modes (4), for the dielectric function lies inside the operator.

In our previous study ${ }^{25}$ we have proposed the interfacial operator approach to compute surface plasmon modes for periodic structures made of dispersive metal, based on the free-electron model. Here, we extend this approach to be applicable to polar materials.

The basic idea is first to deal with the eigensystem (4) in the strict insides of the dielectric and the polar material separately, so that the dielectric function can be moved out of the operator in either region as follows:

$$
-\frac{1}{\varepsilon_{d}} \nabla^{2} H=\Lambda H
$$

$$
-\nabla^{2} H=\varepsilon_{\infty}\left(\frac{\Lambda-\Lambda_{L}}{\Lambda-\Lambda_{T}}\right) \Lambda H,
$$

where $\varepsilon_{d}$ is the dielectric constant of the dielectric material. In the polar material, Eq. (13) is further rearranged in the same manner of Eq. (10) as

$$
\left[\Lambda^{2}-\Lambda\left(\Lambda_{L}-\frac{1}{\varepsilon_{\infty}} \nabla^{2}\right)-\frac{\Lambda_{T}}{\varepsilon_{\infty}} \nabla^{2}\right] H=0 .
$$

Next, consider discretization of Eqs. (12) and (14) in a one-dimensional lattice with the $i$ th point at the interface. The dielectric medium lies to the left of the $i$ th point, the polar material to the right. If we discretize Eqs. (12) and (14) in the strict insides of the dielectric medium and the polar material, respectively, we obtain a system of equation of the form

$$
\mathbf{A}_{(n-1) \times n}(\Lambda) \mathbf{H}_{n}=0,
$$

where $\mathbf{A}$ is an $(n-1) \times n$ matrix, and is quadratic in $\Lambda, \mathbf{H}_{n}$ is the column vector of all discrete $H$ fields, and $\mathbf{H}_{n-1}$ $=\left[H_{1}, \ldots, H_{i-1}, H_{i+1}, \ldots, H_{n}\right]^{T}$ is a subset of $\mathbf{H}_{n}$ excluding $H_{i}$ at the interface. It is obvious that Eq. (15) does not constitute an eigenvalue problem because the matrix $\mathbf{A}$ is not square. One more equation is needed. In fact, Eqs. (12) and (14) are connected by an interface condition

$$
\left[\frac{1}{\varepsilon} \frac{\partial H}{\partial n}\right]_{S}=0
$$

where $\partial / \partial n$ denotes the derivative in the surface normal direction, and $[\cdots]_{S}$ denotes the jump across the interface $S$. The interface condition (16) is obtained by integrating both sides of the eigensystem (4) over a thin box located on the interface, and taking the limit as the box height goes to zero. Applying the dielectric function (1) for the polar material, the interface condition (16) becomes

$$
\left.\frac{1}{\varepsilon_{d}} \frac{\partial H}{\partial n}\right|_{+}=\left.\frac{1}{\varepsilon_{\infty}}\left(\frac{\Lambda-\Lambda_{T}}{\Lambda-\Lambda_{L}}\right) \frac{\partial H}{\partial n}\right|_{-},
$$

where + and - denote the dielectric and the polar material regions, respectively. The next step is to rearrange Eq. (17) as follows:

$$
\left.\frac{\Lambda_{L}}{\varepsilon_{d}} \frac{\partial H}{\partial n}\right|_{+}-\left.\frac{\Lambda_{T}}{\varepsilon_{\infty}} \frac{\partial H}{\partial n}\right|_{-}=\Lambda S,
$$

so that the eigenvalue $\Lambda$ only appears on the right-hand side, where

$$
\left.S \equiv \frac{1}{\varepsilon_{d}} \frac{\partial H}{\partial n}\right|_{+}-\left.\frac{1}{\varepsilon_{\infty}} \frac{\partial H}{\partial n}\right|_{-}
$$

is a weighted difference of the normal derivatives of the $H$ field across the interface.

Apparently, Eqs. (12) and (14), supplemented by Eq. (18), cannot be formulated as a standard eigensystem in terms of $H$, for the right-hand side of Eq. (18) contains the derivatives of $H$. This difficulty can be removed by considering a finitedifference formulation. Recall that Eqs. (12) and (14) have been put in the discretized form, Eq. (15). We will also dis- 
cretize Eq. (18) at the interface point $i$ to yield

$$
\begin{array}{r}
-\frac{\Lambda_{L}}{\varepsilon_{d}} H_{i-1}+\left(\frac{\Lambda_{L}}{\varepsilon_{d}}+\frac{\Lambda_{T}}{\varepsilon_{\infty}}\right) H_{i}-\frac{\Lambda_{T}}{\varepsilon_{\infty}} H_{i+1} \\
=\Lambda\left(\frac{-\varepsilon_{\infty} H_{i-1}+\varepsilon_{\text {sum }} H_{i}-\varepsilon_{d} H_{i+1}}{\varepsilon_{d} \varepsilon_{\infty}}\right),
\end{array}
$$

where $\varepsilon_{\text {sum }}=\varepsilon_{\infty}+\varepsilon_{d}$. The advantage of discretizing Eq. (18) is now clear that the right-hand side of Eq. (20) is simply linear combination of the discrete variables $H_{i-1}, H_{i}$, and $H_{i+1}$. The next key step is to introduce an interfacial variable

$$
R_{i} \equiv \frac{-\varepsilon_{\infty} H_{i-1}+\varepsilon_{\mathrm{sum}} H_{i}-\varepsilon_{d} H_{i+1}}{\varepsilon_{d} \varepsilon_{\infty}}
$$

to replace $H_{i}$ at the interface. Equivalently,

$$
H_{i}=\frac{\varepsilon_{\infty} H_{i-1}+\varepsilon_{d} \varepsilon_{\infty} R_{i}+\varepsilon_{d} H_{i+1}}{\varepsilon_{\text {sum }}} .
$$

Let $\Lambda_{1}=\Lambda_{L}-\Lambda_{T}$ and $\Lambda_{2}=\varepsilon_{\infty} \Lambda_{L}+\varepsilon_{d} \Lambda_{T}$. Substituting Eq. (22) for $H_{i}$ in Eq. (20) yields

$$
\frac{1}{\varepsilon_{\text {sum }}}\left(-\Lambda_{1} H_{i-1}+\Lambda_{2} R_{i}+\Lambda_{1} H_{i+1}\right)=\Lambda R_{i},
$$

which makes the interface variable $R_{i}$ an ideal substitute for $H_{i}$ in formulating the eigenvalue problem. Moreover, we note that $R_{i}$ is a discrete version of $S$ in Eq. (19), and thus a measure of the weighted difference of the normal derivative $\partial H / \partial n$ in two sides of the interface. This important property enables $R_{i}$ to represent the local strength of surface phonon modes at the interface as a surface phonon mode decays rapidly from the interface into both the polar material and the surrounding dielectric.

Finally, all $H_{i}$ appearing in Eq. (15) is replaced by the right-hand side of Eq. (22). Then, Eq. (15) supplemented by Eq. (23) now constitutes a standard quadratic eigenvalue problem of the form

$$
\mathbf{A}_{n \times n}(\Lambda) \widetilde{\mathbf{H}}_{n}=0,
$$

where $\mathbf{A}_{n \times n}$ is a square matrix, and is quadratic in $\Lambda$, and $\widetilde{\mathbf{H}}_{n}$ is $\mathbf{H}_{n}$ with $H_{i}$ replaced by $R_{i}$. Alternatively, Eq. (24) can be recast into a more explicit form

$$
\left(\Lambda^{2}-\Lambda \mathbf{B}-\mathbf{C}\right) \widetilde{\mathbf{H}}=0,
$$

where $\widetilde{\mathbf{H}}=\widetilde{\mathbf{H}}_{n}, \mathbf{B}$ and $\mathbf{C}$ are square matrices. Equation (25) can be written as a linear eigensystem of double size as follows:

$$
\left[\begin{array}{ll}
\mathbf{0} & \mathbf{I} \\
\mathbf{C} & \mathbf{B}
\end{array}\right]\left[\begin{array}{c}
\tilde{\mathbf{H}} \\
\tilde{\mathbf{H}}^{\prime}
\end{array}\right]=\Lambda\left[\begin{array}{c}
\tilde{\mathbf{H}} \\
\tilde{\mathbf{H}}^{\prime}
\end{array}\right],
$$

where $\widetilde{\mathbf{H}}^{\prime}=\Lambda \tilde{\mathbf{H}}$. In the discrete sense, the eigensystem (26) has the same eigenvalue as the original eigensystem (8), although the eigenvector $\widetilde{\mathbf{H}}$ is slightly different from the original eigenvector $\mathbf{H}$. However, they can be converted back and forth between each other through Eqs. (21) and (22). Most importantly, Eq. (26) is a standard eigenvalue problem, and can be solved by many eigenvalue solvers.

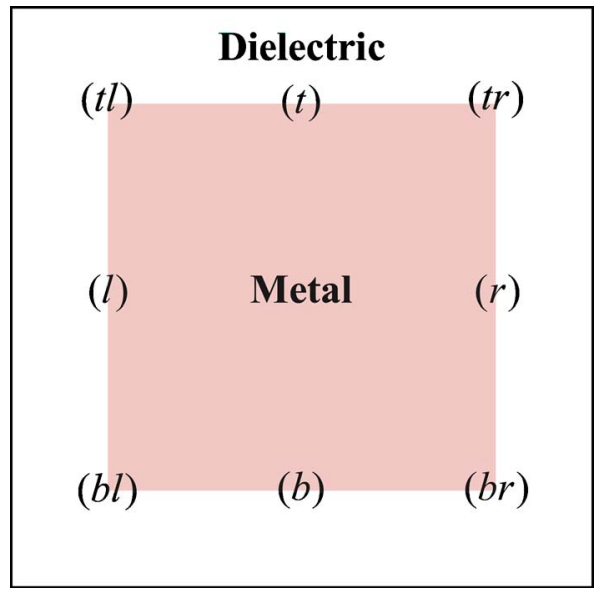

FIG. 2. (Color online) A schematic diagram for the interfacial operator approach in a two-dimensional structure.

The above formulation can be applied to any number of interfaces. One only needs to introduce the same number of interfacial variables.

\section{EXTENSION TO TWO DIMENSIONS}

The method formulated above can be extended to two dimensions in a straightforward manner. However, the details are much more involved than in the one-dimensional (1D) case.

First of all, we discretize the Eqs. (12) and (14) in the strict insides of the dielectric and the polar material by a central finite-difference scheme, respectively. The resulting matrix system is then supplemented by introducing the interfacial variables at the interface between the two media. Figure 2 shows a schematic diagram for the interfacial operator approach in a two-dimensional structure. There are eight types of interface points in 2D. Four types of them appear at sides: left, right, bottom, and top, which are denoted by $(l)$, $(r),(b)$, and $(t)$, respectively, and the other four types appear at corners: bottom left, bottom right, top left and top right, which are denoted by $(b l),(b r),(t l)$, and $(t r)$, respectively. This is due to four different surface normal directions in 2D: vertical, horizontal, and two diagonals, compared to only one direction in 1D. As a result, there are eight types of interfacial variables in 2D defined as follows:

$$
\begin{aligned}
& R_{i, j}^{(l)} \equiv \frac{-\varepsilon_{\infty} H_{i-1, j}+\varepsilon_{\mathrm{sum}} H_{i, j}-\varepsilon_{d} H_{i+1, j}}{\varepsilon_{d} \varepsilon_{\infty}}, \\
& R_{i, j}^{(r)} \equiv \frac{-\varepsilon_{d} H_{i-1, j}+\varepsilon_{\mathrm{sum}} H_{i, j}-\varepsilon_{\infty} H_{i+1, j}}{\varepsilon_{d} \varepsilon_{\infty}}, \\
& R_{i, j}^{(b)} \equiv \frac{-\varepsilon_{\infty} H_{i, j-1}+\varepsilon_{\mathrm{sum}} H_{i, j}-\varepsilon_{d} H_{i, j+1}}{\varepsilon_{d} \varepsilon_{\infty}}, \\
& R_{i, j}^{(t)} \equiv \frac{-\varepsilon_{d} H_{i, j-1}+\varepsilon_{\mathrm{sum}} H_{i, j}-\varepsilon_{\infty} H_{i, j+1}}{\varepsilon_{d} \varepsilon_{\infty}},
\end{aligned}
$$




$$
\begin{aligned}
R_{i, j}^{(b l)} \equiv \frac{-\varepsilon_{\infty} H_{i-1, j-1}+\varepsilon_{\mathrm{sum}} H_{i, j}-\varepsilon_{d} H_{i+1, j+1}}{\varepsilon_{d} \varepsilon_{\infty}} \\
R_{i, j}^{(b r)} \equiv \frac{-\varepsilon_{\infty} H_{i+1, j-1}+\varepsilon_{\mathrm{sum}} H_{i, j}-\varepsilon_{d} H_{i-1, j+1}}{\varepsilon_{d} \varepsilon_{\infty}} \\
R_{i, j}^{(t l)} \equiv \frac{-\varepsilon_{d} H_{i+1, j-1}+\varepsilon_{\mathrm{sum}} H_{i, j}-\varepsilon_{\infty} H_{i-1, j+1}}{\varepsilon_{d} \varepsilon_{\infty}}, \\
R_{i, j}^{(t r)} \equiv \frac{-\varepsilon_{d} H_{i-1, j-1}+\varepsilon_{\mathrm{sum}} H_{i, j}-\varepsilon_{\infty} H_{i+1, j+1}}{\varepsilon_{d} \varepsilon_{\infty}}
\end{aligned}
$$

or, equivalently,

$$
\begin{gathered}
H_{i, j}=\frac{\varepsilon_{\infty} H_{i-1, j}+\varepsilon_{d} \varepsilon_{\infty} R_{i, j}^{(l)}+\varepsilon_{d} H_{i+1, j}}{\varepsilon_{\text {sum }}}, \\
H_{i, j}=\frac{\varepsilon_{d} H_{i-1, j}+\varepsilon_{d} \varepsilon_{\infty} R_{i, j}^{(r)}+\varepsilon_{\infty} H_{i+1, j}}{\varepsilon_{\text {sum }}}, \\
H_{i, j}=\frac{\varepsilon_{\infty} H_{i, j-1}+\varepsilon_{d} \varepsilon_{\infty} R_{i, j}^{(b)}+\varepsilon_{d} H_{i, j+1}}{\varepsilon_{\text {sum }}}, \\
H_{i, j}=\frac{\varepsilon_{d} H_{i, j-1}+\varepsilon_{d} \varepsilon_{\infty} R_{i, j}^{(t)}+\varepsilon_{\infty} H_{i, j+1}}{\varepsilon_{\text {sum }}}, \\
H_{i, j}=\frac{\varepsilon_{\infty} H_{i-1, j-1}+\varepsilon_{d} \varepsilon_{\infty} R_{i, j}^{(b l)}+\varepsilon_{d} H_{i+1, j+1}}{\varepsilon_{\text {sum }}}, \\
H_{i, j}=\frac{\varepsilon_{\infty} H_{i+1, j-1}+\varepsilon_{d} \varepsilon_{\infty} R_{i, j}^{(b r)}+\varepsilon_{d} H_{i-1, j+1}}{\varepsilon_{\text {sum }}}, \\
H_{i, j}=\frac{\varepsilon_{d} H_{i-1, j-1}+\varepsilon_{d} \varepsilon_{\infty} R_{i, j}^{(t r)}+\varepsilon_{\infty} H_{i+1, j+1}}{\varepsilon_{\text {sum }}} . \\
H_{i, j}=\frac{\varepsilon_{d} H_{i+1, j-1}+\varepsilon_{d} \varepsilon_{\infty} R_{i, j}^{(t l)}+\varepsilon_{\infty} H_{i-1, j+1}}{s^{\prime}},
\end{gathered}
$$

Following the same procedure for Eq. (23) in 1D, eight interface conditions in $2 \mathrm{D}$ can be formulated as

$$
\begin{gathered}
\frac{1}{\varepsilon_{\text {sum }}}\left(-\Lambda_{1} H_{i-1, j}+\Lambda_{2} R_{i, j}^{(l)}+\Lambda_{1} H_{i+1, j}\right)=\Lambda R_{i, j}^{(l)}, \\
\frac{1}{\varepsilon_{\text {sum }}}\left(\Lambda_{1} H_{i-1, j}+\Lambda_{2} R_{i, j}^{(r)}-\Lambda_{1} H_{i+1, j}\right)=\Lambda R_{i, j}^{(r)}, \\
\frac{1}{\varepsilon_{\text {sum }}}\left(-\Lambda_{1} H_{i, j-1}+\Lambda_{2} R_{i, j}^{(b)}+\Lambda_{1} H_{i, j+1}\right)=\Lambda R_{i, j}^{(b)}, \\
\frac{1}{\varepsilon_{\text {sum }}}\left(\Lambda_{1} H_{i, j-1}+\Lambda_{2} R_{i, j}^{(t)}-\Lambda_{1} H_{i, j+1}\right)=\Lambda R_{i, j}^{(t)}, \\
\frac{1}{\varepsilon_{\text {sum }}}\left(-\Lambda_{1} H_{i-1, j-1}+\Lambda_{2} R_{i, j}^{(b l)}+\Lambda_{1} H_{i+1, j+1}\right)=\Lambda R_{i, j}^{(b l)},
\end{gathered}
$$

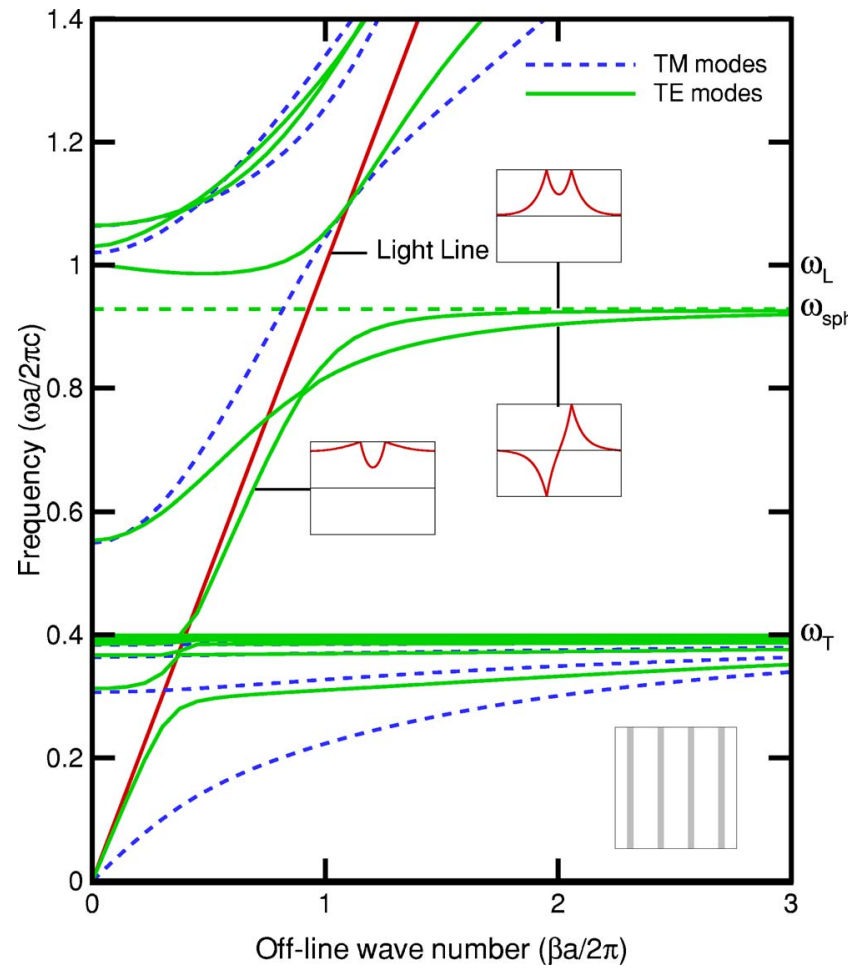

FIG. 3. (Color online) The dispersion relations at $k=0$ for a one-dimensional polaritonic layered structure [Fig. 1(a)] of thickness $t / a=0.2$, where $\omega_{T} a / 2 \pi c=0.4, \omega_{L} a / 2 \pi c=1$, and $\varepsilon_{\infty}=5.1$.

$$
\begin{aligned}
& \frac{1}{\varepsilon_{\text {sum }}}\left(-\Lambda_{1} H_{i+1, j-1}+\Lambda_{2} R_{i, j}^{(b r)}+\Lambda_{1} H_{i-1, j+1}\right)=\Lambda R_{i, j}^{(b r)}, \\
& \frac{1}{\varepsilon_{\text {sum }}}\left(\Lambda_{1} H_{i+1, j-1}+\Lambda_{2} R_{i, j}^{(t l)}-\Lambda_{1} H_{i-1, j+1}\right)=\Lambda R_{i, j}^{(t l)}, \\
& \frac{1}{\varepsilon_{\text {sum }}}\left(\Lambda_{1} H_{i-1, j-1}+\Lambda_{2} R_{i, j}^{(t r)}-\Lambda_{1} H_{i+1, j+1}\right)=\Lambda R_{i, j}^{(t r)} .
\end{aligned}
$$

As to the strict insides of the dielectric and the polar material, Eqs. (12) and (14) are discretized to give

$$
\begin{gathered}
\Lambda^{2} H_{i, j}+\Lambda\left(\frac{1}{\varepsilon_{d} h^{2}} L_{i, j}\right)=0, \\
\Lambda^{2} H_{i, j}+\Lambda\left(\frac{1}{\varepsilon_{\infty} h^{2}} L_{i, j}-\Lambda_{L} H_{i, j}\right)-\frac{\Lambda_{T}}{\mu_{\infty} h^{2}} L_{i, j}=0,
\end{gathered}
$$

where $L_{i, j}=H_{i-1, j}+H_{i, j-1}-4 H_{i, j}+H_{i+1, j}+H_{i, j+1}$.

Second, since there are four neighbor points instead of two, incorporated in the discretization of the $\nabla^{2}$ operator in 2D, replacing $H_{i, j}$ at the interface in Eqs. (30) and (31) with $R_{i j}$ through Eq. (28) becomes more complicated in 2D. However, this could be done in a systematic and efficient way. Equations (29) to (31) are combined together to form a quadratic eigensystem in the same form of Eq. (25), which in turn can be written as a linear eigensystem as in Eq. (26). 


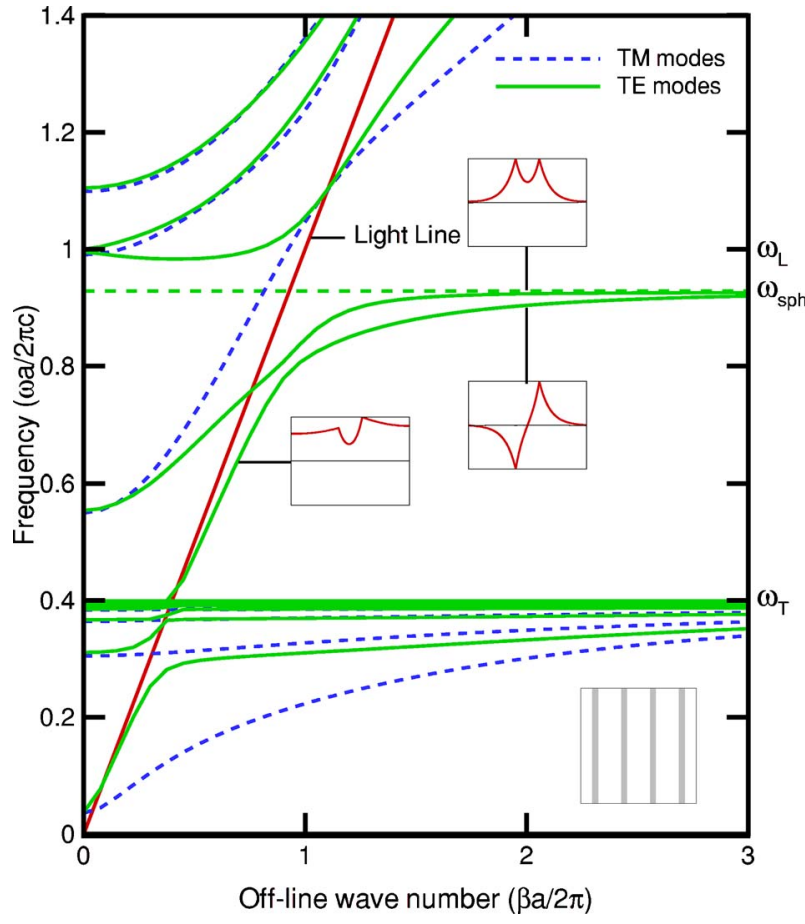

FIG. 4. (Color online) The dispersion relations at $k a / 2 \pi=0.1$ for a one-dimensional polaritonic layered structure [Fig. 1(a)] of thickness $t / a=0.2$, where $\omega_{T} a / 2 \pi c=0.4, \omega_{L} a / 2 \pi c=1$, and $\varepsilon_{\infty}$ $=5.1$.

\section{RESULTS AND DISCUSSION}

In the present study, we consider the polar material $\mathrm{TlCl}$ used in Ref. 21, where $\varepsilon_{\infty}=5.1, \omega_{T} a / 2 \pi c=0.4$, and $\omega_{L} a / 2 \pi c=1.0$ for the lattice constant $a=10 \mu \mathrm{m}$. For $\varepsilon_{d}=1$, we have $\omega_{s p h} a / 2 \pi c=0.9286$, according to Eq. (2). First of all, we will study the one-dimensional crystal in Fig. 1(a), and then proceed with the study of two dimensional crystals in Figs. 1(b)-1(d).

\section{A. 1D array of polar materials}

For the one-dimensional layered structures [Fig. 1(a)], there are two surface phonon modes inside the polariton gap $\left(\omega_{T}<\omega<\omega_{L}\right)$ for the TE bands. One has a lower frequency with odd symmetry and the other has a higher frequency with even symmetry. Figure 3 shows the dispersion relations at the zone center $(k=0)$ for the thickness ratio $t / a=0.2$. The insets show the eigenmodes of two TE bands at the off-line wave number $\beta a / 2 \pi=2$, and of the lower band at $\beta a / 2 \pi$ $=0.7$, where $\beta$ is the wave number parallel to the interface. Note that a crossing scheme is observed between the two TE bands in the polariton gap for $k=0$. Near the crossing point, usually at a small off-line wave number, the mode of the lower TE band switches from even symmetry to odd symmetry. In addition, we see an anticrossing scheme between the two TE bands inside the polariton gap for $k \neq 0$. Figure 4 shows the dispersion relations at $k a / 2 \pi=0.1$ for the same structure in Fig. 3. In this case, the eigenmodes of the two TE bands no longer possess perfectly different symmetries of odd and even at small off-line wave numbers. This is due to

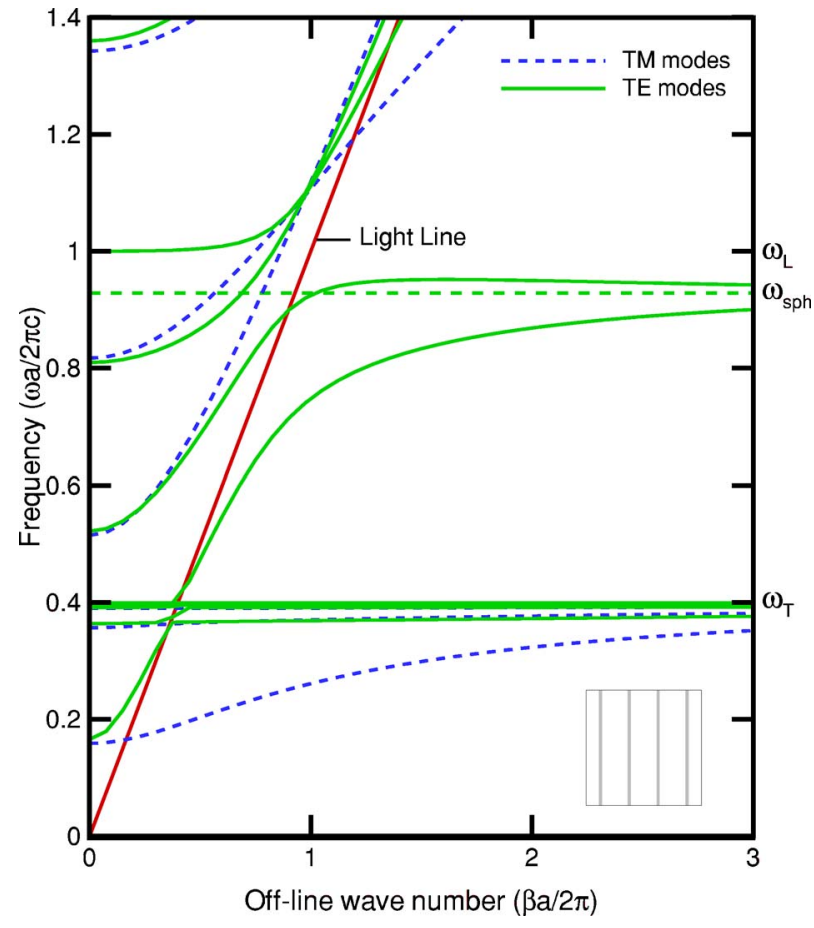

FIG. 5. (Color online) The dispersion relations at $k a / 2 \pi=0.5$ for a one-dimensional polaritonic layered structure [Fig. 1(a)] of thickness $t / a=0.1$, where $\omega_{T} a / 2 \pi c=0.4, \omega_{L} a / 2 \pi c=1$, and $\varepsilon_{\infty}$ $=5.1$.

the phase difference $e^{i k a}$ of the modes between the two sides of the unit cell, as can be seen from the Bloch condition (6). In view of the similar symmetries of the modes, no intersections can be found between the two TE bands. ${ }^{26}$ However, they eventually grow into even and odd modes at large offline wave numbers, for the fields at the unit cell boundary become very small due to the evanescent nature of surface phonon modes.

Splitting of the modes comes from interaction of surface phonons on both sides of the polar material as well as the dielectric. The mode with even symmetry has a higher frequency because the mode structure has a larger area that effectively corresponds to a larger energy. At sufficiently large off-line wave numbers, the frequencies of two surface phonon modes converge to the same frequency $\omega_{\text {sph }}$ given in Eq. (2). For a very thin structure, convergence of surface phonon modes is slow. This is due to effective interaction of the modes from both sides of the polar material, which lifts the degeneracy. Figure 5 shows the dispersion relations at $k a / 2 \pi=0.5$ for the thickness ratio $t / a=0.1$. In a range of medium fractions of the polar material, convergence of surface phonon modes becomes faster. However, for a very high filling fraction, convergence is slow again, for the degeneracy is again lifted by effective interaction of the modes from both sides of the dielectric. Figure 6 shows the dispersion relations at the zone edge $(k a / 2 \pi=0.5)$ for the thickness ratio $t / a=0.9$. In the meanwhile, the higher TE band inside the polariton gap has a higher frequency that approaches the LO phonon frequency $\omega_{L}$ at zero off-line wave number. This is reasonable for the whole lattice is almost filled with the polar material. Another important fact in Fig. 6 is the nega- 


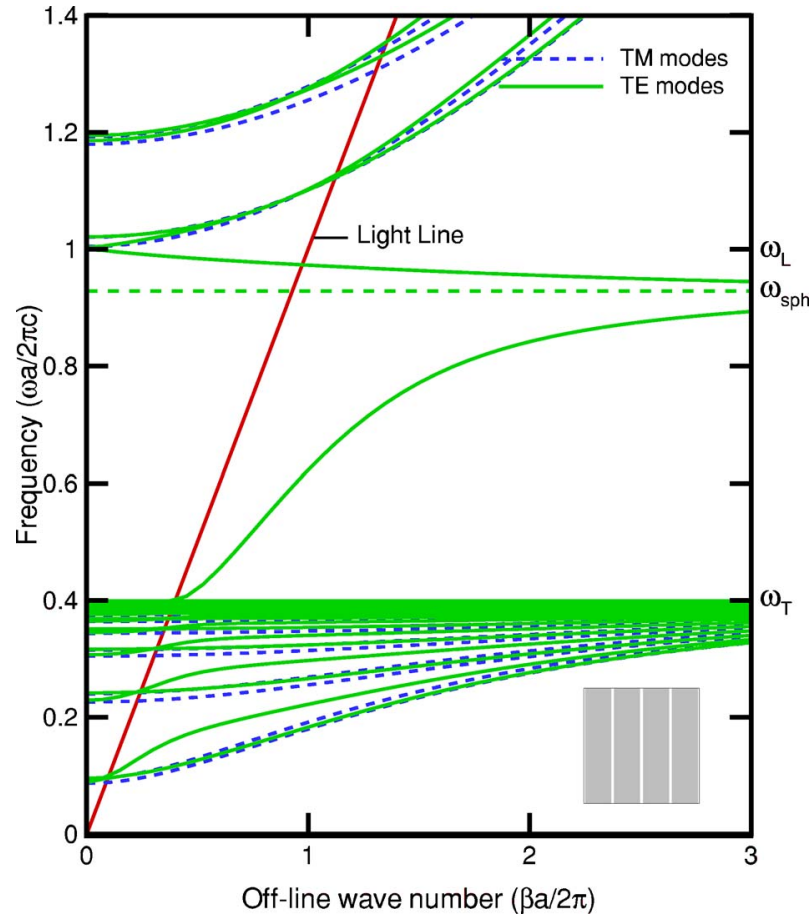

FIG. 6. (Color online) The dispersion relations at $k a / 2 \pi=0.5$ for a one-dimensional polaritonic layered structure [Fig. 1(a)] of thickness $t / a=0.9$, where $\omega_{T} a / 2 \pi c=0.4, \omega_{L} a / 2 \pi c=1$, and $\varepsilon_{\infty}$ $=5.1$.

tive group velocity of the higher TE band, which occurs as the dielectric portion becomes sufficiently small. This is consistent with the property of left handedness for the waveguide stack in Ref. 27, which serves as an approach to making a material with a negative index of refraction. Figure 6 also shows that as the thickness ratio $t / a$ is close to 1 , all the frequency bands (bulk modes) are expelled from the polariton gap, except the two TE bands (surface modes) which converge to $\omega_{\text {sph }}$ from below and above, respectively, at large off-line wave numbers.

In addition to surface phonon modes inside the polariton gap, a large number of nearly dispersionless bands for both TM and TE modes intensively gather around the TO phonon frequency $\omega_{T}$ from below. They are resonant cavities modes ${ }^{28}$ which correspond to very large values of dielectric constant. Figure 6 further shows that the bands of resonant cavity modes are more spread if the thickness ratio $t / a$ is large. This can be explained by examining the waveguide modes in high- $\varepsilon$ cylinders, and we will come back to this point in the discussion for two-dimensional arrays of polar materials.

\section{B. 2D arrays of polar materials}

In order to ensure the accuracy of the eigenfrequencies, computations are performed on five different grids. Table I lists the numerics of $\omega_{2}$ and $\omega_{48}$ for TE modes at the zone center $\Gamma$ for a square array of square cylinders in Fig. 1(b). Here, $\omega_{2}$ is the first nonzero eigenfrequency, and $\omega_{48}$ is the 48th eigenfrequency which is close to the TO phonon frequency $\omega_{T}$. The computed results show good agreements be-
TABLE I. Convergence test for the eigenfrequency against the grid size.

\begin{tabular}{ccccc}
\hline \hline$N_{\text {grid }}$ & $20^{2}$ & $30^{2}$ & $40^{2}$ & $50^{2}$ \\
\hline$\omega_{2} a / 2 \pi c$ & 0.2580 & 0.2583 & 0.2585 & 0.2585 \\
$\omega_{48} a / 2 \pi c$ & 0.3887 & 0.3895 & 0.3906 & 0.3911 \\
\hline \hline
\end{tabular}

tween different grid resolutions. Figure 7 also shows the distribution of eigenfrequencies for the same structure. The frequencies are little dependent on the grid level except in two distinct regions: one below the TO phonon frequency $\omega_{T}$ (resonant cavity modes) and one around the surface phonon frequency $\omega_{\text {sph }}$ (surface phonon modes). As expected, the modes intensively distributed below $\omega_{T}$ are the resonant cavity modes, which we have also observed in one-dimensional layered structures. However, contrary to the one-dimensional polaritonic crystals which have only two branches of surface phonon modes, the two-dimensional polaritonic crystals apparently have infinite degrees of surface phonon mode gathering around the surface phonon frequency $\omega_{\text {sph. }}$. The same figure appears to indicate that the number of resolved surface phonon modes (resonant cavity modes) increases linearly (quadratically) with the number of grid points.

In order to see the fuller details, we plot the bands of TM and TE modes separately in Figs. 8 and 9 for a square array of circular cylinders of radius $r / a=0.3$ in Fig. 1(c). The same figures also show the bands of the metallodielectric crystal for comparison, where polar materials are replaced by perfect conductors. Both polarizations (TM and TE) have resonant cavity modes. However, only one polarization (TE) has surface phonon modes. These two types of modes (surface phonon modes and resonant cavity modes) come from different physical origins. For frequencies immediately below $\omega_{T}$, there is large dielectric constant which can support infinite degrees of resonant cavity modes. On the other hand, surface phonon modes come from the coupling between the electromagnetic wave and vibration of the ionic charge of the polar

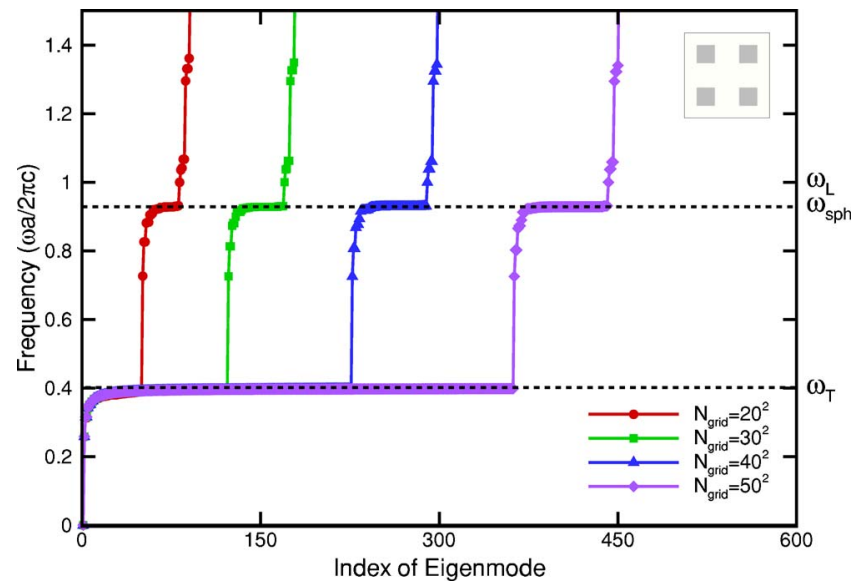

FIG. 7. (Color online) The eigenfrequencies for TE modes versus the index of eigenmode computed with different grid resolutions at the point $\Gamma$ for a square array of square cylinders [Fig. 1(b)] of half width $w / a=0.2$ where $\omega_{T} a / 2 \pi c=0.4, \omega_{L} a / 2 \pi c=1$, and $\varepsilon_{\infty}=5.1$. 


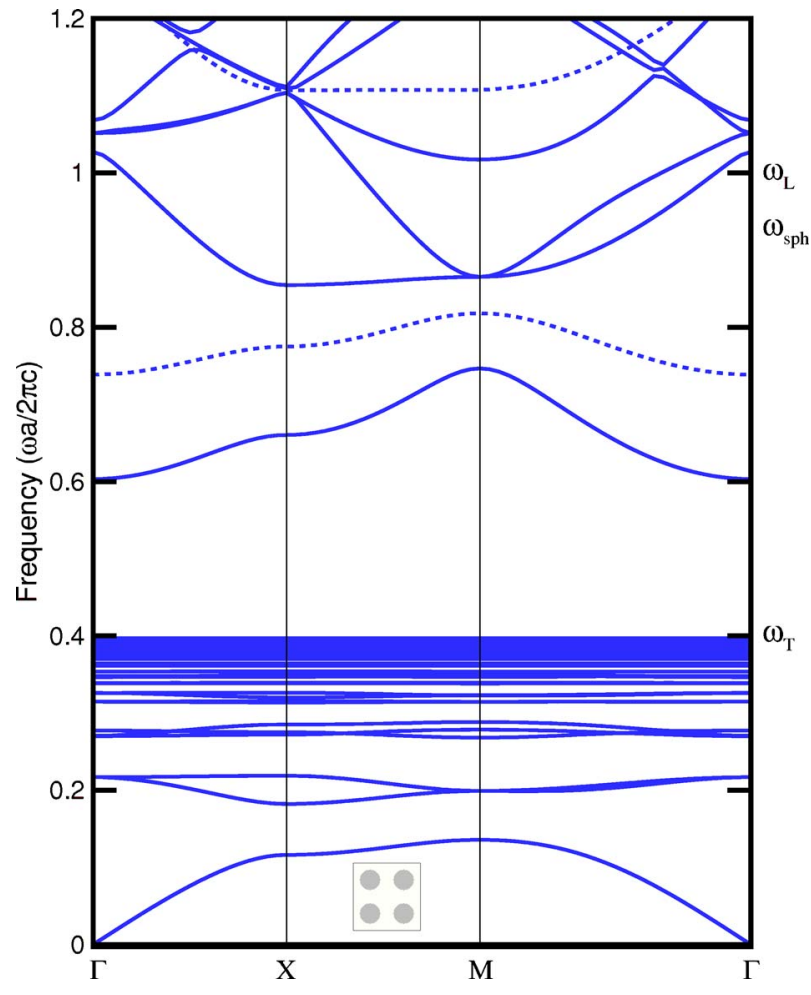

FIG. 8. (Color online) The TM band structure for a square array of circular cylinders [Fig. 1(c)] of radius $r / a=0.3$, where $\omega_{T} a / 2 \pi c=0.4, \omega_{L} a / 2 \pi c=1$, and $\varepsilon_{\infty}=5.1$. The dashed lines denote the bands of metallodielectric crystals obtained by replacing the polar material with a perfect metal.

material, which is not allowed for TM modes.

If $\omega_{T}$ goes down to zero, resonant cavity modes would disappear, and $\omega_{L}$ would act similar to $\omega_{p}$ (plasma frequency in the free-electron model for dispersive metals). If also $\omega_{L}$ goes to infinity, the frequency of surface phonon modes would go to infinity as well, and all the other bands would converge upward to the bands of the metallodielectric crystals. This upward convergence can be explained by considering the Rayleigh quotient for TE modes (also for TM modes)

$$
R_{H}=\frac{\langle H, \mathcal{L} H\rangle}{\langle H, H\rangle},
$$

where $\langle f, g\rangle=\int_{V_{\text {cell }}} f^{*} g d \tau$ denotes the inner product of $f$ and $g$ over the unit cell $V_{\text {cell }}$. Each eigenfrequency is obtained by minimizing the Rayleigh quotient with respect to functions which are orthogonal to all the lower frequency modes. Note that perfect conductors expel fields completely. The metallodielectric crystal therefore has less freedom in distributing the energy in the unit cell, and has higher eigenfrequencies compared to the arrays of polar materials which allow limited energy distribution in themselves. Below, we shall focus on discussion of resonant cavity modes and surface phonon modes separately.

\section{Resonant cavity modes}

Important physics related to resonant cavity modes have been investigated in depth in Ref. 28, such as anticrossing

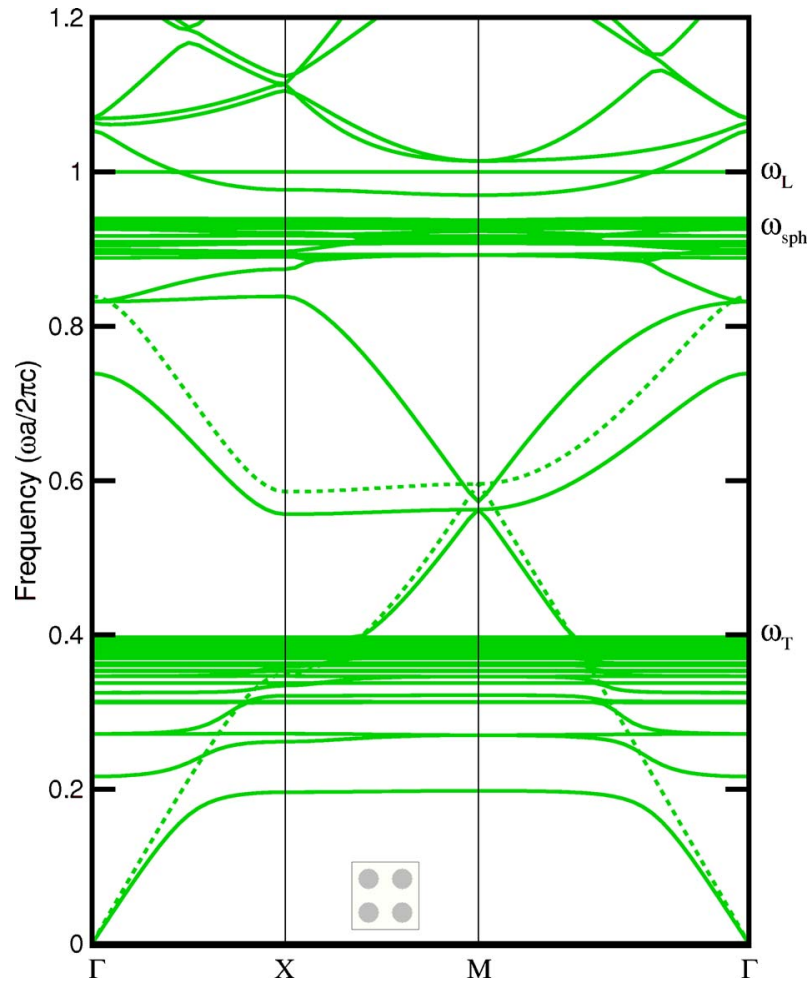

FIG. 9. (Color online) The TE band structure for a square array of circular cylinders [Fig. 1(c)] of radius $r / a=0.3$ where $\omega_{T} a / 2 \pi c=0.4, \omega_{L} a / 2 \pi c=1$, and $\varepsilon_{\infty}=5.1$. The dashed lines denote the bands of metallodielectric crystals obtained by replacing the polar material with a perfect metal.

interaction of the TE bands with the metallodielectric bands, node switching from one pattern to another, flux expulsion with small changes in frequency across the TO phonon frequency $\omega_{T}$, and so forth. Although resonant cavity modes have similar dispersionless characteristic as surface phonon modes, they are bulk modes in nature, and the number of which is even larger than that of surface phonon modes. From Fig. 7, we see that the number of stationary modes around the TO phonon frequency $\omega_{T}$ increases quadratically with the grid resolution. This is consistent with the resonances of the square cavity to the metallic waveguide modes with frequencies $\omega_{l m}$ determined by two free indices $l$ and $m^{28}$

$$
\omega_{l m}=\frac{\pi c}{2 w \sqrt{\varepsilon_{l m}}} \sqrt{l^{2}+m^{2}},
$$

where $\varepsilon_{l m}=\varepsilon_{\infty}\left(\omega_{l m}^{2}-\omega_{L}^{2}\right) /\left(\omega_{l m}^{2}-\omega_{T}^{2}\right)$. Solving Eq. (33) for $\omega_{l m}$ yields $^{28}$

$$
\omega_{l m}^{2}=\frac{2 \Omega_{l m}^{2} \omega_{T}^{2}}{\omega_{L}^{2}+\Omega_{l m}^{2}+\sqrt{\left(\omega_{L}^{2}+\Omega_{l m}^{2}\right)^{2}-4 \Omega_{l m}^{2} \omega_{T}^{2}}},
$$

where $\Omega_{l m}=\pi c \sqrt{l^{2}+m^{2}} / 2 w \sqrt{\boldsymbol{\epsilon}_{\infty}}$ with $w$ the half width of the square cavity. The expression (34) indicates $\omega_{T}$ is the upper limit frequency of resonant cavity modes for if $\Omega_{l m}$ goes to infinity, $\omega_{l m}$ approaches $\omega_{T}$. On the other hand, if $\Omega_{l m}$ goes to $0, \omega_{l m}$ approaches $\Omega_{l m} \omega_{T} / \omega_{L}$. Therefore, for large fraction of 


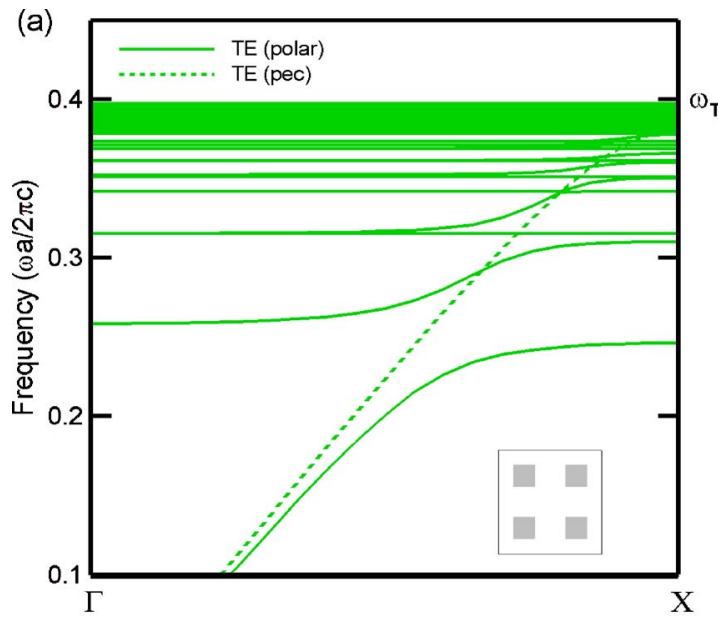

(b)

(c)
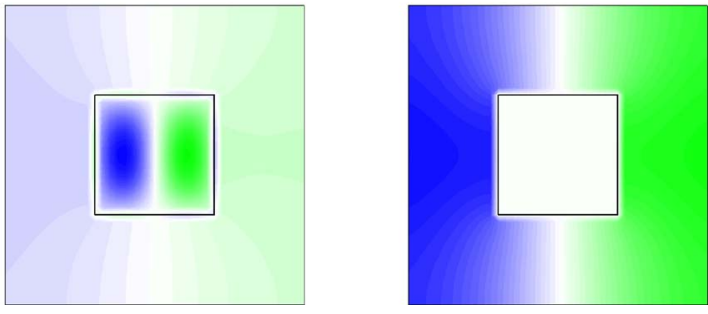

FIG. 10. (Color online) (a) The TE band structure for a square array of square cylinders [Fig. 1(b)] of half width $w / a=0.2$ where $\omega_{T} a / 2 \pi c=0.4, \omega_{L} a / 2 \pi c=1$, and $\varepsilon_{\infty}=5.1$. (b) The real part of the eigenmode for the second polaritonic band at $k a / 2 \pi=0.4$. (c) Same as (b) for the first metallodielectric band.

polar materials, that is, large value of $w / a$, the resonant bands spread more widely.

As the frequency approaches $\omega_{T}$ from below, the bands become more concentrated. All these bands are flattened except an anticrossing interaction with the bands of metallodielectric crystals (by replacing the polar material with a perfect metal), in particular, for TE modes. The anticrossing interaction is present only for modes with even symmetry with respect to the wave vector, and is possible due to small but finite leakage of the modes out of the polar material, ${ }^{28}$ while the field for the metallodielectric structure is completely compelled from the perfect metal. In Fig. 10, we plot the portion of the TE bands along the $\Gamma-X$ path for a square array of square cylinders of half width $w / a=0.2$, along with the real part of the magnetic field of the eigenmodes for the second polaritonic band and the first metallodielectric band at $k a / 2 \pi=0.4$, which is near the anticrossing point. We can observe the leakage of the magnetic field out of the polar material in Fig. 10(b), with a contrast to the completely expelled field out of the perfect metal in Fig. 10(c). The overlap integral of the two modes serves as an indication of the anticrossing interaction. ${ }^{28}$ Therefore, we define an anticrossing index $\eta$ for the two modes $f$ and $g$ as

$$
\eta=\frac{|\langle f, g\rangle|^{2}}{\langle f, f\rangle\langle g, g\rangle} .
$$

For two modes with different symmetries, this value is zero and there are no anticrossing schemes, while for two modes

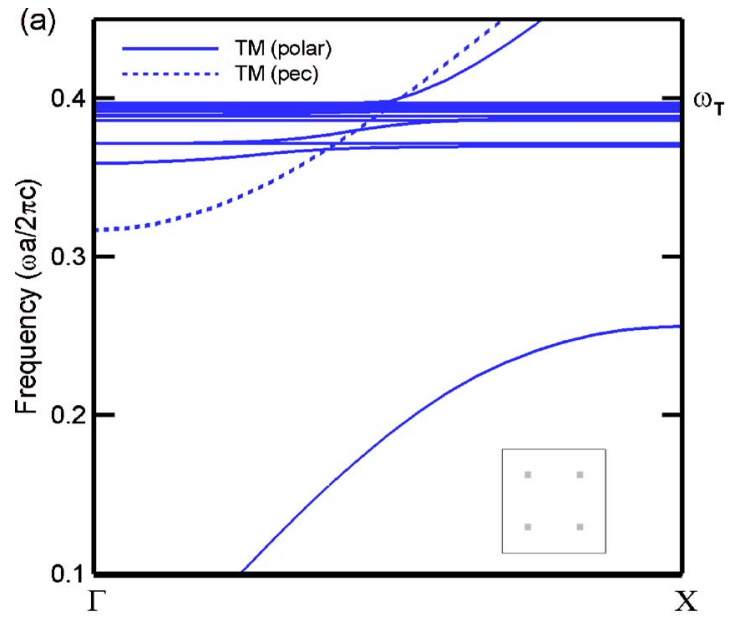

(b)

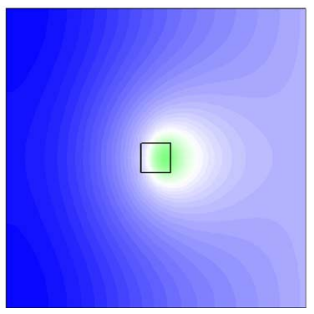

(c)

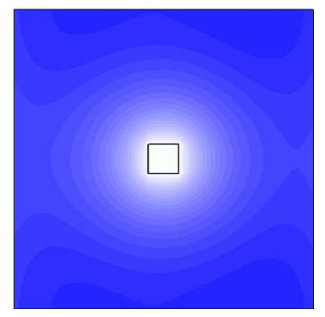

FIG. 11. (Color online) (a) The TM band structure for a square array of square cylinders [Fig. 1(b)] of half width $w / a=0.05$, where $\omega_{T} a / 2 \pi c=0.4, \omega_{L} a / 2 \pi c=1$, and $\varepsilon_{\infty}=5.1$. (b) The real part of the eigenmode for the second polaritonic band at $k a / 2 \pi=0.2$. (c) Same as (b) for the first metallodielectric band.

with like symmetries, this value is always larger than zero and an anticrossing scheme can be observed. In this case, for $f$ and $g$ correspond to the modes in Figs. 10(b) and 10(c), respectively, the anticrossing index $\eta=0.277$.

In Ref. 28, the anticrossing interaction occurs only for TE modes, for the TM metallodielectric band usually has a cutoff frequency higher than the chosen TO phonon frequency $\omega_{T}$ in normalized unit, and thus no anticrossing interaction was found for TM modes. However, the anticrossing interaction can also be observed for TM modes if the cutoff frequency is small enough such that the metallodielectric band penetrates through the resonant bands. A lower cutoff frequency for the corresponding TM metallodielectric band can be obtained with a smaller filling fraction of the polar material. In Fig. 11, we plot the portion of the TM bands along the $\Gamma-X$ path for a square array of square cylinders of half width $w / a=0.05$, along with the real part of the electric field of the eigenmodes for the second polaritonic band and the first metallodielectric band at $k a / 2 \pi=0.2$, which is near the anticrossing point. In this case, the two modes in Figs. 11(b) and $11(\mathrm{c})$ has an anticrossing index $\eta=0.835$, which is substantially larger than that for Figs. 10(b) and 10(c). This is due to more leakage of the electric field out of the polar material for TM modes in Fig. 11(b), which can be explained on a unified basis by examining different types of boundary conditions for TM and TE modes, respectively. For TE modes, the interface condition $\left[\frac{1}{\varepsilon} \frac{\partial H}{\partial n}\right]_{S}$ allows a drastic change of the magnetic field across the interface $S$ between 
(a)

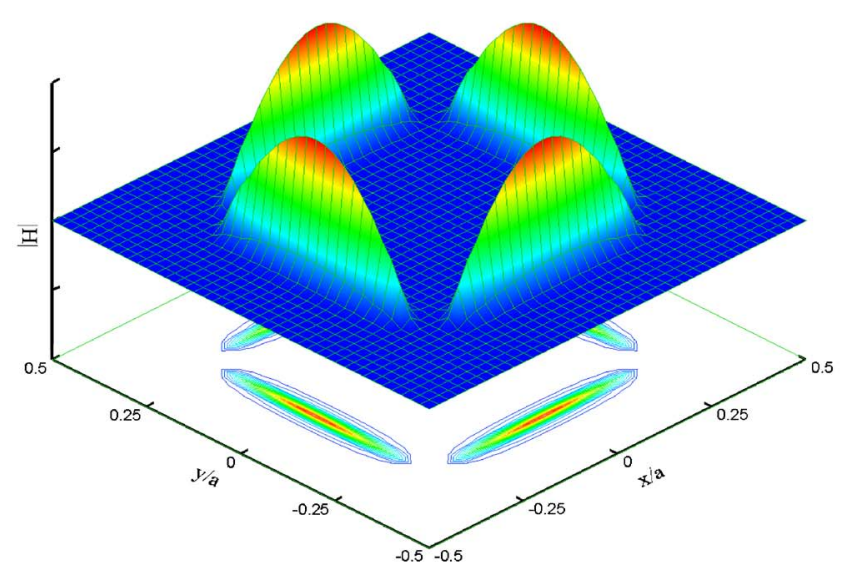

(b)

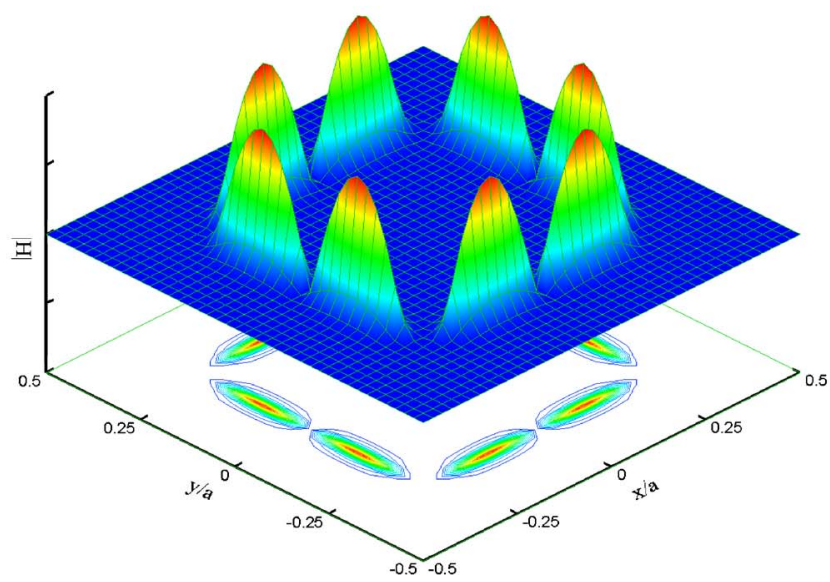

(c)

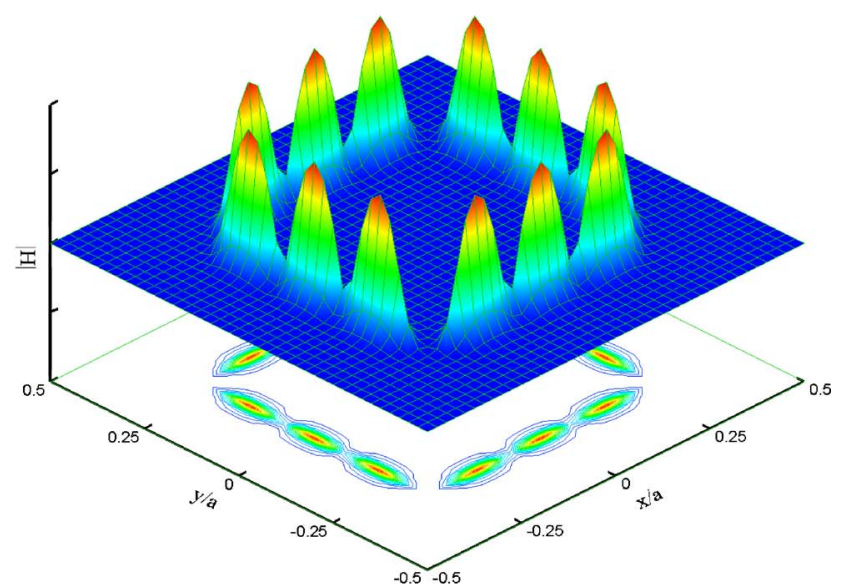

(d)

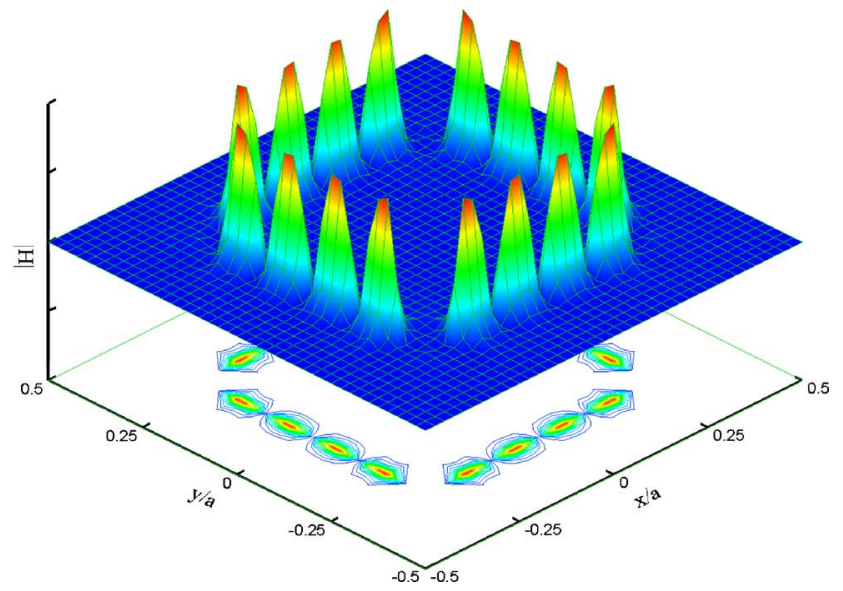

FIG. 12. (Color online) The $H$ field in magnitude for four typical surface phonon modes with $\omega a / 2 \pi c=0.9286$ at the point $\Gamma$ near the surface phonon frequency $\omega_{s p h}$ for a square array of square metallic cylinders [Fig. 1(b)] of half width $w / a=0.3$, where $\omega_{T} a / 2 \pi c=0.4$, $\omega_{L} a / 2 \pi c=1$, and $\varepsilon_{\infty}=5.1$.

the dielectric and the polar material, which may prevent a large leakage of the field from the polar material to the dielectric. On the contrary, for TM modes, the interface condition $\left[\frac{\partial E}{\partial n}\right]_{S}$ has to be satisfied at the interface $S$. Continuity of the normal derivative of the electric field prevents a drastic change of the electric field across the interface, and results in a larger leakage of the field out of the polar material. More leakage of the electric field gives rise to a larger value of the overlap integral, which also means that the anticrossing behavior is stronger. However, due to the cutoff behavior of the metallodielectric band, the anticrossing scheme is not so typical as for TE modes.

\section{Surface phonon modes}

For the one-dimensional layered structures [Fig. 1(a)], there are only two surface phonon modes with odd and even symmetries, and the offline wave number is essential to provide the momentum along the interface and sustain surface phonon modes. For the two-dimensional structures [Figs. $1(\mathrm{~b})$ and $1(\mathrm{~d})]$, there are as many surface phonon modes as possible, and the off-plane wave number is not necessary to sustain surface phonon modes. This is because the two- dimensional structure has a continuous interface which allows a degree of freedom to sustain the mode oscillation. Figure 12 shows the $H$ field in magnitude for four typical surface phonon modes at the point $\Gamma$ near the surface phonon frequency $\omega_{\text {sph }}$ for a square array of square cylinders of half width $w / a=0.3$. Note that in all the plots of the TE eigenmodes, the $H$ field is normalized to have maximum unity, that is, $|H|_{\max }=1$. Surface phonon modes may be as sharp as a knife edge living on the interface. With the interfacial operator approach, it only takes a few points to resolve this feature. Note that in Fig. 12 the typical feature of the surface phonon modes is similar except a different variation along the interface. However, the eigenfrequency is almost identical.

Apparently variation of the $H$ field along the interface between the polar material and the dielectric does not alter the value of the eigenfrequency, and the interface can sustain as many stationary modes as it could. Consequently, there are expected to be infinite number of surface phonon modes around $\omega_{\mathrm{sph}}$, analogous to the case of surface plasmon modes. ${ }^{14,25}$ The highly degenerate nature and infinite number of surface phonon modes can be further explained through the Rayleigh quotient $R_{H}$ (32). It is known that the eigenfre- 
quency corresponds to minimization of the Rayleigh quotient under a constraint that the corresponding eigenfunction be orthogonal to all previously obtained eigenfunctions. For a linear operator $\mathcal{L}$, Eq. (32) can be used directly, while for a nonlinear operator, the Rayleigh quotient $R_{H}$ has to be obtained in a slightly different manner. Based on the interfacial operator approach developed in the previous section, we rewrite the eigensystems (12) and (14) in the dielectric and the polar material regions, respectively, as

$$
\begin{gathered}
\Lambda^{2} H+\Lambda\left(\frac{1}{\varepsilon_{d}} \nabla^{2} H\right)=0, \\
\Lambda^{2} H-\Lambda\left(\Lambda_{L} H-\frac{1}{\varepsilon_{\infty}} \nabla^{2} H\right)-\frac{\Lambda_{T}}{\varepsilon_{\infty}} \nabla^{2} H=0 .
\end{gathered}
$$

Taking inner product of $H$ with each term of the above two equations, adding them together, performing the integration by parts, and using the Bloch condition (6), we obtain a quadratic expression for the Rayleigh quotient $R_{H}$ :

$$
R_{H}^{2} A-R_{H} B+C=0
$$

or, equivalently,

$$
R_{H}=\frac{B \pm \sqrt{B^{2}-4 A C}}{2 A}
$$

where

$$
\begin{gathered}
A=\int_{V_{\text {cell }}}|H|^{2} d \tau, \\
B=\int_{S_{m}} H^{*} S d a+\frac{1}{\varepsilon_{d}} \int_{V_{d}}|\nabla H|^{2} d \tau+\int_{V_{m}}\left(\frac{1}{\varepsilon_{\infty}}|\nabla H|^{2}\right. \\
\left.+\Lambda_{L}|H|^{2}\right) d \tau, \\
C=-\left.\frac{\Lambda_{T}}{\varepsilon_{\infty}} \int_{S_{m}} H^{*} \frac{\partial H}{\partial n}\right|_{-} d a+\frac{\Lambda_{T}}{\varepsilon_{\infty}} \int_{V_{m}}|\nabla H|^{2} d \tau,
\end{gathered}
$$

with $V_{d}$ and $V_{m}$ denoting the volumes of the dielectric and the polar material, respectively, of the unit cell, and $S_{m}$ the surface of $V_{m}$. In the expression of $B, S$ appears in the surface integral term, which accounts for the contribution of the strength of surface phonon mode to the eigenfrequency. Note also that only the normal derivative of the $H$ field occurs in the surface integral, and the tangential variation of the $H$ field will not change the value of $R_{H}$ as well as the eigenfrequency. There can be as many modes as possible if the variation of the $H$ field in the normal direction to the interface remains unchanged. This can be verified from the field patterns of the typical surface phonon modes in Fig. 12, which shows different degrees of oscillation along the interface. All the four modes have the same frequency $\omega a / 2 \pi c=0.9286$ with three significant digits. Surface phonon modes of higher oscillation can be resolved only when the grid resolution is fine enough to tell the tangential variation.

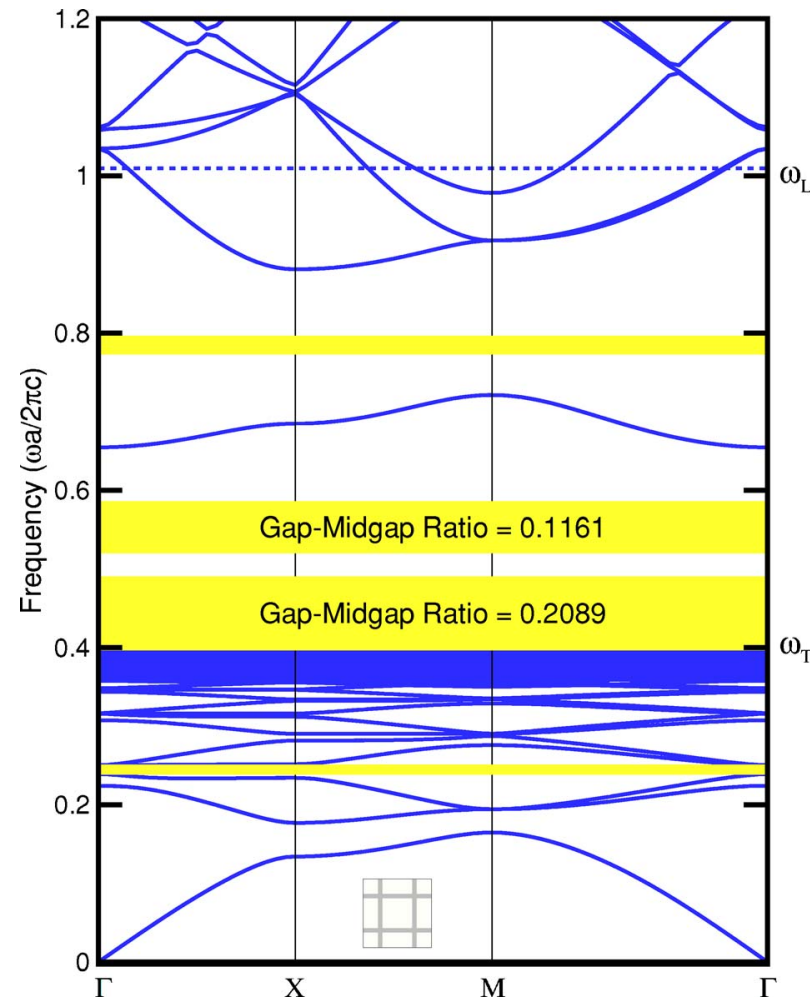

FIG. 13. (Color online) The TM band structure for a square array of grid cylinders [Fig. 1(d)] of thickness $t / a=0.1$ where $\omega_{T} a / 2 \pi c=0.4, \omega_{L} a / 2 \pi c=1$, and $\varepsilon_{\infty}=5.1$. The dashed lines denote the metallodielectric bands obtained by replacing the polar material with a perfect metal.

Another important aspect of surface phonon modes is band flattening and band broadening. The band flattening for the two-dimensional structures [Figs. 1(b)-1(d)] is due to strong phonon-photon coupling that reduces the band dispersion. ${ }^{19}$ Basically, band flattening occurs for frequency bands around the surface phonon frequency $\omega_{\text {sph }}$ and resonant cavity modes below the TO phonon frequency $\omega_{T}$. However, the shape/geometry of the polar material is a major factor in determining the overall band pattern. Figures 13 and 14 show the band structures for a square array of grid cylinders of thickness $t / a=0.1$ [Fig. 1(d)]. In particular, Fig. 14 shows that the flattened bands gathering around the surface phonon frequency $\omega_{\text {sph }}$ spread more widely compared to those of circular cylinders in Fig. 9. This is the phenomenon of band broadening, which is similar to the behavior of surface phonon modes for plasmonic crystals. ${ }^{25}$ This band broadening is due to effective interaction of the modes on both sides of the polar material, which lifts the degeneracy. Moreover, the overall band structure for the grid cylinders exhibits a general flattening tendency, thus opening up wide photonic band gaps within the polariton gap $\left(\omega_{T}<\omega<\omega_{L}\right)$. As a comparison, Fig. 9 for round-shaped circular cylinders, shows that the frequency bands inside the polariton gap extends widely in frequency, blocking the full gap region and denying opening up of photonic band gaps. On the other hand, the bands of resonant cavity modes for grid cylinders become more concentrated near $\omega_{T}$ because thin-striped polar materials allow less freedom in distributing the fields of 


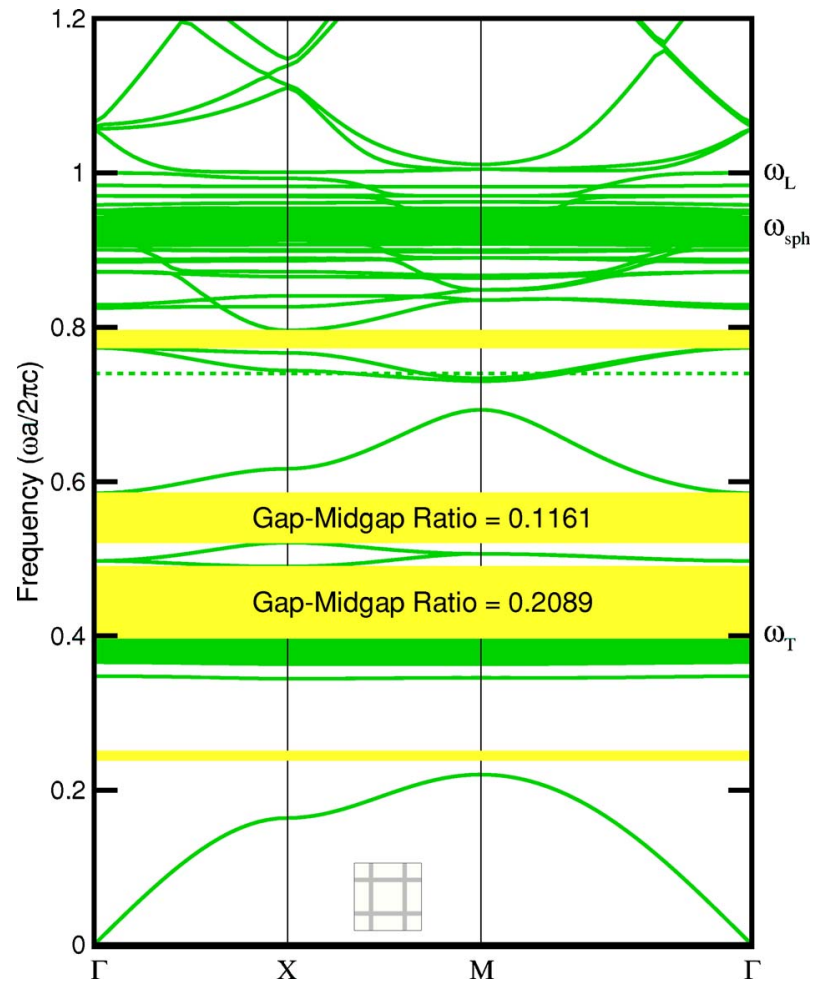

FIG. 14. (Color online) The TE band structure for a square array of grid cylinders [Fig. 1(d)] of thickness $t / a=0.1$, where $\omega_{T} a / 2 \pi c=0.4, \omega_{L} a / 2 \pi c=1$, and $\varepsilon_{\infty}=5.1$. The dashed lines denote the metallodielectric bands obtained by replacing the polar material with a perfect metal.

bulk modes. If the thickness ratio $t / a$ or $r / a$ is large, surface phonon modes are more densely distributed in frequency, while resonant cavity modes spread more widely.

\section{Longitudinal modes}

The electromagnetic fields are transverse in nature. However, longitudinal modes may exist in a material when the dielectric constant becomes zero. According to the dielectric function (1) for polar materials, a longitudinal mode exists when its eigenfrequency is equal to the LO phonon frequency $\omega_{L}$. That also means oscillation of the electric field coincides with the coherent motion of the electrons. As with surface phonon modes, longitudinal modes appear only in the TE modes, for the transversality condition of the $E$ field $(\boldsymbol{\nabla} \cdot \mathbf{E}=\mathbf{0})$ is always met for the TM modes. However, longitudinal modes are difficult to obtain due to singularity of the operator in Eq. (4). Nevertheless, with rearrangement of the interfacial operator approach in Eq. (4), based on the dielectric function (1), the singularity is removed and longitudinal modes can be solved. Figure 15 shows the static mode at the point $\Gamma$ for a square array of circular cylinders of radius $r / a=0.3$. Note that the longitudinal mode is constant in the metal, which is the typical feature of longitudinal oscillation.

\section{CONCLUDING REMARKS}

In this paper, we proposed the interfacial operator approach to compute band structures of polaritonic crystals or

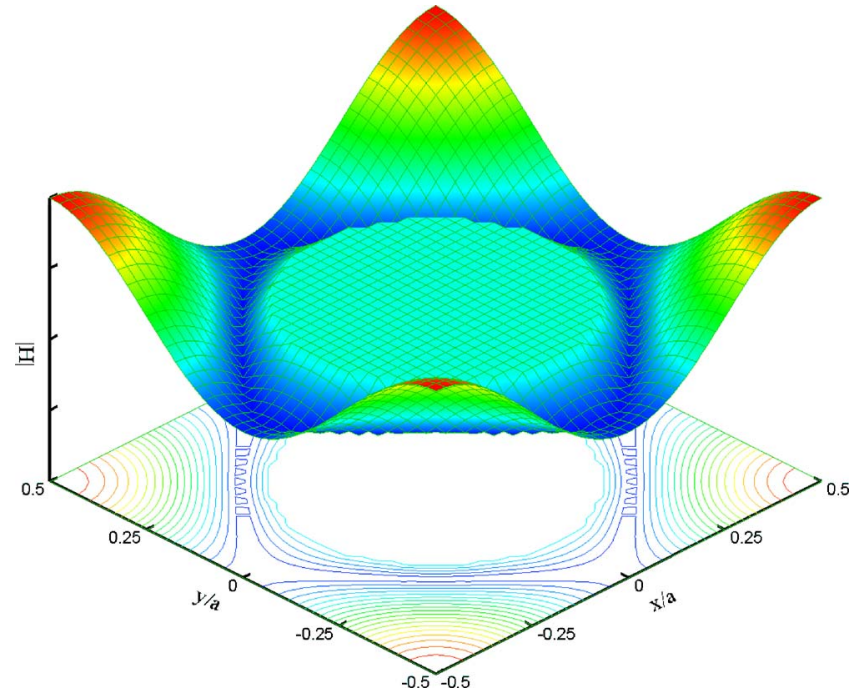

FIG. 15. (Color online) The longitudinal mode at the point $\Gamma$ for a square array of circular cylinders [Fig. 1(c)] of radius $r / a=0.3$ where $\omega_{T} a / 2 \pi c=0.4, \omega_{L} a / 2 \pi c=1$, and $\varepsilon_{\infty}=5.1$.

photonic crystals of polar materials in one and two dimensions. In particular, an interfacial variable is introduced to measure the weighted difference of the normal derivatives of the $H$ field across the interface, and thus accounts for the local strength of the surface phonon modes. Fine resolution at different grid levels shows that the mode frequencies are not very dependent upon the number of grid points except for the possible infinite degeneracy of surface phonon modes and the many resonant cavity modes. The number of resolved resonant cavity modes and surface phonon modes do depend on the grid resolution.

The method has been applied to study four types of photonic crystals of polar materials. In particular, we have examined the effects of dimension, the size (filling ratio) effect, the effect of the intrinsic frequencies $\omega_{T}$ (the transverse optical phonon frequency), and $\omega_{L}$ (the longitudinal optical phonon frequency) as well as the geometric (shape) effect of the polar material. Physical details have been discussed regarding the crossing and anticrossing schemes of band dispersion, distribution of resonant cavity modes, localized nature of surface phonon modes, lifting degeneracy by thinning polar materials, and the limiting behaviors of the band structures at the small limit of $\omega_{T}$ and large limit of $\omega_{L}$. Several interesting features were uncovered and explained and the main results were summarized in the Introduction. As a final remark, we have not considered the effect of dissipation of polaritonic structures which is substantial in the infrared regime. The issue is now under investigation, and the results will be reported elsewhere.

\section{ACKNOWLEDGMENTS}

This work was supported in part by National Science Council of the Republic of China under Contract Nos. NSC 94-2212-E-002-047 and NSC 94-2212-E-002-076, and the Ministry of Economic Affairs of the Republic of China under Contract No. MOEA 94-EC-17-A-08-S1-0006. 
*Electronic address: chern@iam.ntu.edu.tw

†Electronic address: mechang@gate.sinica.edu.tw

${ }^{1}$ E. Yablonovitch, Phys. Rev. Lett. 58, 2059 (1987).

${ }^{2}$ S. John, Phys. Rev. Lett. 58, 2486 (1987).

${ }^{3}$ D. R. Smith, S. Schultz, N. Kroll, M. Sigalas, K. M. Ho, and C. M. Soukoulis, Appl. Phys. Lett. 65, 645 (1994).

${ }^{4}$ N. A. Nicorovici, R. C. McPhedran, and L. C. Botten, Phys. Rev. E 52, 1135 (1995).

${ }^{5}$ S. Fan, P. R. Villeneuve, and J. D. Joannopoulos, Phys. Rev. B 54, 11245 (1996).

${ }^{6}$ T. Suzuki and P. K. L. Yu, Phys. Rev. B 57, 2229 (1998).

${ }^{7}$ A. Moroz, Phys. Rev. B 66, 115109 (2002).

${ }^{8}$ E. I. Smirnova, C. Chen, M. A. Shapiro, J. R. Sirigiri, and R. J. Temkin, J. Appl. Phys. 91, 960 (2002).

${ }^{9}$ C. C. Chang, J. Y. Chi, R. L. Chern, C. C. Chang, C. H. Lin, and C. O. Chang, Phys. Rev. B 70, 075108 (2004).

${ }^{10}$ A. R. McGurn and A. A. Maradudin, Phys. Rev. B 48, 17576 (1993).

${ }^{11}$ V. Kuzmiak, A. A. Maradudin, and F. Pincemin, Phys. Rev. B 50, 16835 (1994).

${ }^{12}$ M. M. Sigalas, C. T. Chan, K. M. Ho, and C. M. Soukoulis, Phys. Rev. B 52, 11744 (1995).

${ }^{13}$ I. El-Kady, M. M. Sigalas, R. Biswas, K. M. Ho, and C. M. Soukoulis, Phys. Rev. B 62, 15299 (2000).

${ }^{14}$ T. Ito and K. Sakoda, Phys. Rev. B 64, 045117 (2001).

${ }^{15}$ E. Moreno, D. Erni, and C. Hafner, Phys. Rev. B 65, 155120
(2002).

${ }^{16}$ I. I. Smolyaninov, W. Atia, and C. C. Davis, Phys. Rev. B 59, 2454 (1999).

${ }^{17}$ G. Shvets and Y. A. Urzhumov, J. Opt. A, Pure Appl. Opt. 7, S23 (2005).

${ }^{18}$ M. M. Sigalas, C. M. Soukoulis, C. T. Chan, and K. M. Ho, Phys. Rev. B 49, 11080 (1994).

${ }^{19}$ W. Zhang, A. Hu, X. Lei, N. Xu, and N. Ming, Phys. Rev. B 54, 10280 (1996).

${ }^{20}$ V. Kuzmiak, A. A. Maradudin, and A. R. McGurn, Phys. Rev. B 55, 4298 (1997).

${ }^{21}$ K. C. Huang, P. Bienstman, J. D. Joannopoulos, K. A. Nelson, and S. Fan, Phys. Rev. Lett. 90, 196402 (2003).

${ }^{22}$ C. Kittel, Introduction to Solid State Physics, 7th ed. (John Wiley \& Sons, New York, 1996).

${ }^{23}$ O. Toader and S. John, Phys. Rev. E 70, 046605 (2004).

${ }^{24}$ A. Modinos, N. Stefanou, and V. L. Yannopapas, Opt. Express 8, 197 (2001)

${ }^{25}$ C. C. Chang, R. L. Chern, C. C. Chang, and R. R. Hwang, Phys. Rev. B 72, 205112 (2005).

${ }^{26}$ L. D. Landau and E. M. Lifshitz, Quantum Mechanics (Pergamon, New York, 1977).

${ }^{27}$ G. Shvets, Phys. Rev. B 67, 035109 (2003).

${ }^{28}$ K. C. Huang, P. Bienstman, J. D. Joannopoulos, K. A. Nelson, and S. Fan, Phys. Rev. B 68, 075209 (2003). 